\title{
Development and internal validation of prediction models for colorectal cancer survivors to estimate the 1-year risk of low health-related quality of life in multiple domains
}

Dóra Révész ${ }^{1,2^{*}}$ (D), Sander M. J. van Kuijk ${ }^{3}$, Floortje Mols ${ }^{2,4}$, Fränzel J. B. van Duijnhoven ${ }^{5}$, Renate M. Winkels ${ }^{6}$, Huub Hoofs ${ }^{7}$, I Jmert Kant ${ }^{7}$, Luc J. Smits ${ }^{7}$, Stéphanie O. Breukink ${ }^{8}$, Lonneke V. van de Poll-Franse ${ }^{3,4,9}$, Ellen Kampman ${ }^{5}$, Sandra Beijer ${ }^{4}$, Matty P. Weijenberg ${ }^{1}$ and Martijn J. L. Bours ${ }^{1}$

\begin{abstract}
Background: Many colorectal cancer (CRC) survivors experience persisting health problems post-treatment that compromise their health-related quality of life (HRQOL). Prediction models are useful tools for identifying survivors at risk of low HRQoL in the future and for taking preventive action. Therefore, we developed prediction models for CRC survivors to estimate the 1-year risk of low HRQoL in multiple domains.

Methods: In 1458 CRC survivors, seven HRQoL domains (EORTC QLQ-C30: global QoL; cognitive, emotional, physical, role, social functioning; fatigue) were measured prospectively at study baseline and 1 year later. For each HRQoL domain, scores at 1-year follow-up were dichotomized into low versus normal/high. Separate multivariable logistic prediction models including biopsychosocial predictors measured at baseline were developed for the seven HRQoL domains, and internally validated using bootstrapping.

Results: Average time since diagnosis was 5 years at study baseline. Prediction models included both nonmodifiable predictors (age, sex, socio-economic status, time since diagnosis, tumor stage, chemotherapy, radiotherapy, stoma, micturition, chemotherapy-related, stoma-related and gastrointestinal complaints, comorbidities, social inhibition/negative affectivity, and working status) and modifiable predictors (body mass index, physical activity, smoking, meat consumption, anxiety/depression, pain, and baseline fatigue and HRQoL scores). Internally validated models showed good calibration and discrimination (AUCs: 0.83-0.93).

(Continued on next page)
\end{abstract}

\footnotetext{
* Correspondence: Dora.Revesz@maastrichtuniversity.nl; D.Revesz@uvt.nl

'Department of Epidemiology, GROW - School for Oncology and

Developmental Biology, Maastricht University, P. Debyeplein 1, 6200, MD,

Maastricht, the Netherlands

${ }^{2}$ Department of Medical and Clinical Psychology, CoRPS - Center of Research

on Psychology in Somatic diseases, Tilburg University, Warandelaan 2, 5037,

$A B$, Tilburg, the Netherlands

Full list of author information is available at the end of the article
}

(c) The Author(s). 2020 Open Access This article is licensed under a Creative Commons Attribution 4.0 International License, which permits use, sharing, adaptation, distribution and reproduction in any medium or format, as long as you give appropriate credit to the original author(s) and the source, provide a link to the Creative Commons licence, and indicate if changes were made. The images or other third party material in this article are included in the article's Creative Commons licence, unless indicated otherwise in a credit line to the material. If material is not included in the article's Creative Commons licence and your intended use is not permitted by statutory regulation or exceeds the permitted use, you will need to obtain permission directly from the copyright holder. To view a copy of this licence, visit http://creativecommons.org/licenses/by/4.0/ The Creative Commons Public Domain Dedication waiver (http://creativecommons.org/publicdomain/zero/1.0/) applies to the data made available in this article, unless otherwise stated in a credit line to the data. 
(Continued from previous page)

Conclusions: The prediction models performed well for estimating 1-year risk of low HRQoL in seven domains. External validation is needed before models can be applied in practice.

Keywords: Colorectal cancer, Cancer survivors, Quality of life, Prediction models, Model development, Internal validation

\section{Background}

The number of colorectal cancer (CRC) survivors is increasing as a result of rising incidence rates related to population ageing and a more widespread adoption of western lifestyles and of rising survival rates due to improved treatments and implementation of screening programs [1-3]. CRC survivors are often not only concerned about how long they will survive after treatment (quantity of life) but also how well they will survive (quality of life), because after diagnosis and treatment many survivors continue to experience physical and psychosocial problems and long-lasting and late treatment effects that can have a major impact on their healthrelated quality of life (HRQoL) [2, 4-6]. To anticipate the occurrence of potential HRQoL problems and enable appropriate preventive actions, it is important to identify individual survivors who have an increased risk of experiencing HRQoL problems in the future. Estimation of the future risk of low HRQoL in multiple domains, such as global quality of life and several functioning domains (e.g. physical, social and role functioning), can offer opportunities for tailoring of appropriate preventive interventions aimed at safeguarding the HRQoL of CRC survivors, for example through health behavioral interventions [7-13]. However, tools for risk estimation of future HRQoL are currently not available for CRC survivors.

In order to identify CRC survivors at risk of having low HRQoL in the future, accurate risk estimation must be based on relevant predictive factors incorporated in risk prediction models. Previous studies have investigated associations of clinical, personal, lifestyle, and psychosocial factors with HRQoL in CRC survivors [14-16]. Although such research enhances our understanding of the disease and treatments effects on HRQoL, it remains to be investigated whether these factors are useful for risk estimation. No study has yet incorporated these factors into risk prediction models, which are statistical models that enable estimation of the risk of some outcome variable based on a collection of predictors that should be interpreted in combination and not in isolation [17]. Several models have been developed to predict overall or progression-free survival after CRC, both using clinical and comorbidity factors, thereby aiding the decision-making process regarding treatment choices for individual CRC patients [18-21]. Up to date, however, no models have been developed for predicting future HRQoL in CRC survivors, whilst such prognostic models could be invaluable for identifying individuals at risk of future low HRQoL, preferably in multiple domains to estimate personal risk profiles that can indicate future problems in specific HRQoL domains [22-24].

Risk prediction models should be developed and rigorously tested according to a systematic research approach $[25,26]$. Prediction research generally consists of three successive steps: 1 . model development and internal validation, 2. external model validation, and 3. clinical impact evaluation. Development of a prediction model should always start with an evidence-based selection of candidate predictors potentially eligible for inclusion in an appropriate statistical model $[17,25,26]$. As starting point for developing a prediction model for HRQoL of CRC survivors, we have therefore provided a broad overview of candidate predictors of HRQoL in CRC survivors in a systematic review [27]. Using the World Health Organization's International Classification of Functioning, Disability and Health (WHO-ICF) as guiding framework, candidate predictors were mapped across relevant biopsychosocial domains of health and functioning and classified according to their strength of evidence [27]. The systematic review served as evidence base for selecting relevant candidate predictors to be used for the initial development of risk prediction models for HRQoL in CRC survivors. Models should preferably also be internally validated during the model development phase, which means testing the initial model for reproducibility $[17,25,26]$. Subsequently, during the second step of prediction research, the predictive performance of newly developed and internally validated models needs to be evaluated in populations other than the population used for model development (external validation) to assess the generalizability of prediction models $[25,26]$. Finally, before implementation of prediction models in clinical practice, the presentation (e.g. as a risk score) and clinical impact of externally validated models should ideally be evaluated by testing whether their application in practice leads to improved patient outcomes, such as HRQoL $[25,26]$.

In the present study, as a first step towards use of risk prediction models for HRQoL in oncology practice, multivariable prediction models to estimate the 1-year risk of low HRQoL in multiple domains were developed and 
internally validated in a large prospective cohort of longterm CRC survivors. We primarily aimed to develop well-performing internally valid prognostic models for separate HRQoL domains, based on a comprehensive set of evidence-based a priori defined biopsychosocial predictors. A secondary goal was to build models that are easy for clinical practice, and can be used to prevent low future $\mathrm{HRQoL}$ in at-risk CRC survivors.

\section{Methods}

\section{Study population}

Data was used of stage I-IV CRC survivors participating in a prospective cohort study within the Patient Reported Outcomes Following Initial Treatment and LongTerm Evaluation of Survivorship (PROFILES) registry [28]. PROFILES is linked to the Netherlands Cancer Registry that routinely collects information from all newly diagnosed cancer patients in The Netherlands. The study was conducted according to the Declaration of Helsinki guidelines and approved by a certified local medical ethics committee, and written informed consent was obtained from all subjects before participation. Details of the data collection have previously been reported [28]. In short, CRC survivors participating in the prospective cohort study were asked to complete surveys with self-administered questionnaires, either online or on paper, in yearly waves from 2010 onwards. For the present analyses, we used data from three consecutive waves conducted between 2012 and 2014. Data from the first two waves (T0 and T1), which for individual participants was completed within a period of approximately 6 months, was considered as study baseline and used for assessment of candidate predictors. Data from the third wave (T2), which was completed for individual participants approximately 1 year after the first wave, was considered as follow-up for prediction of HRQoL. More details and timing of the three waves are shown in Fig. 1. All subjects who responded at the first wave (T0) were included in the present analyses $(N=1458)$.

\section{Data collection \\ Health-related quality of life}

HRQoL was measured at T0 and T2 with the European Organization for Research and Treatment of Cancer Quality of life Questionnaire - Core 30 (EORTC QLQC30, Version 3.0) [29]. Seven subscales of this validated cancer-specific questionnaire were used for assessing the following HRQoL domains: global QoL; cognitive, emotional, physical, role, and social functioning; and fatigue. For every subscale a sum score was calculated ranging from 0 to 100 points, with higher scores on the global QoL and functioning scales representing better HRQoL and functioning, and higher scores on the fatigue scale representing worse fatigue [29]. Our goal was to develop prediction models for estimating the risk of having low HRQoL at follow-up (T2). Since interpretation of an individual's continuous score on one or more of the HRQoL subscales of the EORTC QLQ-C30 is difficult in regard to risk prediction, the scores of the separate HRQoL subscales were dichotomized into low vs. nor$\mathrm{mal} / \mathrm{high}$ scores for the purpose of developing the prediction models to estimate the risk of low HRQoL. Cutoffs to dichotomize the subscale scores of the separate HRQoL domains were determined based on previously published medium-to-large minimally important deteriorations (MID) in the EORTC QLQ-C30 subscales [30]. Accordingly, individuals were classified as having low HRQoL within each domain when having a subscale score at $\mathrm{T} 2 \geq 1$ MID below the group average subscale score at T0; otherwise they were classified as having nor$\mathrm{mal} /$ high HRQoL. In this way, the low HRQoL group was comprised of individuals who either reported a constantly low HRQoL score at both T0 and T2, or who experienced a clinically relevant deterioration from a normal/high HRQoL score at T0 to a low HRQoL score at T2 (Table 1).

\section{Candidate predictors}

Using our previously published biopsychosocial WHOICF framework [27], a comprehensive set of sociodemographic, clinical, lifestyle, and psychological factors was selected as candidate predictors, including both nonmodifiable and modifiable variables (see Supplementary Figure 1). The majority of candidate predictors was measured at the first wave (T0), except for certain lifestyle factors that were measured in a subsequent wave approximately 6 months later (T1).

Sociodemographic factors Sociodemographic predictors included age, sex, current marital status (married or cohabiting, yes/no), and current work status (yes/no). Socio-economic status (SES) was categorized into low, medium or high, based on individual fiscal data from the year 2000 on the economic value of homes and household incomes, aggregated per postal code [31].

Clinical factors Comorbidities were assessed with the adjusted Self-Administered Comorbidity Questionnaire (SCQ) [32], and categorized into 0,1 , or $\geq 2$ comorbidities. Clinical data related to the patient's history of CRC included the date of diagnosis, tumor site (colon or rectum), tumor stage (I-IV), and treatments received in addition to surgery (chemotherapy and/or radiotherapy). The presence of a stoma was assessed with the CRCspecific CR38 module of the EORTC QLQ [33].

Symptom scales and single items of the EORTC QLQC30 and CR38 were used to assess cancer-related symptoms, including fatigue, stoma-related complaints (for 


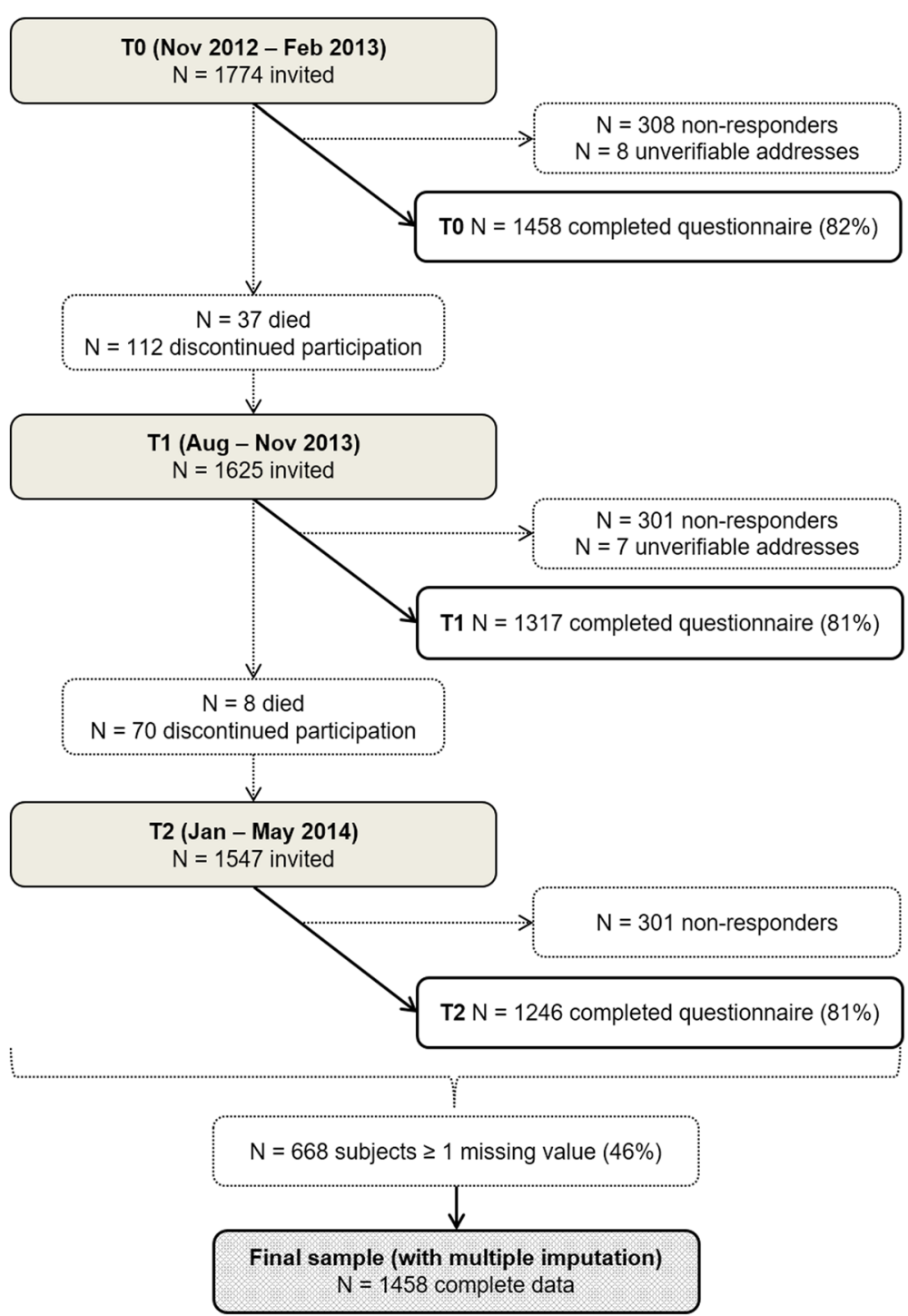

Fig. 1 Flowchart of participant recruitment and inclusion at three consecutive waves (TO, T1 and T2) of the prospective cohort study in colorectal cancer survivors from the PROFILES registry

persons without a stoma, missing values were imputed with a '0' for 'no complaints'), pain, micturition, and chemotherapy-related side effects. Baseline fatigue scores were entered into all models as predictor based on strong evidence for its relevance as a HRQoL predictor $[27,34]$. The separate subscale scores of nausea/vomiting, constipation, diarrhea, defecation problems, and gastrointestinal problems were summed into a total score for 'gastrointestinal symptoms'.

Lifestyle factors As measures of body fatness, body mass index $\left(\mathrm{BMI}, \mathrm{kg} / \mathrm{m}^{2}\right)$ was calculated from selfreported height and weight at T0, and self-assessed waist circumference $(\mathrm{cm})$ at $\mathrm{T} 1$. Current smoking status $(\mathrm{y} / \mathrm{n})$ was assessed by self-report at T0, whereas alcohol consumption, physical activity, and fruit, vegetable and total meat consumption were collected at $\mathrm{T} 1$ by validated questionnaires. Based on the 2007 World Cancer Research Fund/American Institute for Cancer Research (WCRF/AICR) lifestyle recommendations [35], participants were categorized into non-drinkers, mild-moderate drinkers ( $\leq 1$ drinks/day for women and $\leq 2$ drinks/day for men), or heavy drinkers ( $>1$ drink/day for women and $>2$ drinks/day for men). Physical activity was assessed by the Short QUestionnaire to ASsess Health-enhancing physical activity (SQUASH) [36]. Total time spent in moderate-tovigorous intensity physical activity (MVPA, min/day) was calculated [36, 37], on the basis of which adherence $(y / n)$ to the Dutch physical activity standard was determined (i.e. MVPA $\geq 30 \mathrm{~min} /$ day on $\geq 5$ days/week). Dietary intake 
Table 1 Health-related quality of life (HRQOL) domains at baseline and follow-up of the entire study population in the non-imputed dataset $(N=1458)$

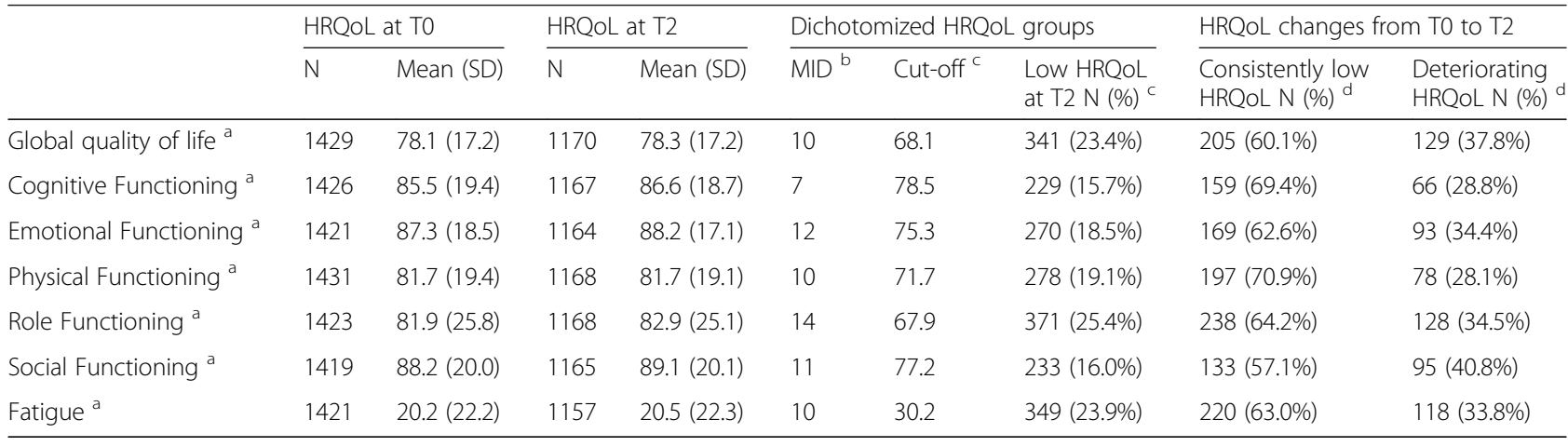

Footnotes:

${ }^{a}$ Higher scores on global QoL and functioning domains represent better HRQoL, whereas higher scores on fatigue represent worse fatigue complaints. All domains scores can range from 0 to 100 points

${ }^{\mathrm{b}}$ Minimal important deterioration (MID) in EORTC OLQ-C30 domains published by Cocks et al. for each subscale [18]

'Persons were classified as having 'low HRQoL' when their T2 score differed by $\geq 1$ MID from the T0 group mean (below mean for global quality of life and functioning domains; above mean for fatigue)

${ }^{\mathrm{d}}$ Persons had consistently low HRQoL when both their T0 and T2 scores were $\geq 1 \mathrm{MID}$ above/below the T0 group mean. Persons had deteriorating HRQoL when they decreased from normal/high HRQoL at T0 to a T2 score $\geq 1$ MID below/above the T0 group mean (below mean for global quality of life and functioning domains; above mean for fatigue). The number of subjects with consistently low and deteriorating HRQoL do not add up to the total number in the low HRQoL group due to missings at TO

was measured by an adapted version of the Dutch Healthy Diet-Food Frequency Questionnaire (DHD-FFQ) [38]. Adherence to the 2007 WCRF/AICR guidelines regarding fruit and vegetable intake and meat consumption [35] was defined as eating $\geq 5$ portions of fruits and/or vegetables each day $(\mathrm{y} / \mathrm{n})$ and eating $<5$ portions of meat per week $(\mathrm{y} / \mathrm{n})$.

Psychological factors Separate scores for anxiety and depressive symptoms were calculated from the Hospital Anxiety and Depression Scale (HADS, range: 0-21 points), with higher scores indicating more symptoms [39]. Subscales of the Dutch 14-item Type D Personality Scale (DS14) [40] were used to assess 'Negative Affectivity' (i.e. the tendency to experience negative emotions) and 'Social Inhibition' (i.e. the tendency to inhibit expression of emotions in social interaction) [41].

\section{Statistical analyses}

Prior to analyses, incomplete data on candidate predictors and HRQoL outcomes was imputed with $50 \mathrm{mul}$ tiple imputations using predictive mean matching in the mice package in $\mathrm{R}$ [42]. Multivariable logistic regression analyses were performed to develop separate prediction models for the seven HRQoL domains in the rms package in R [43]. Based on the previously developed WHOICF framework [27], 12 factors for which strong evidence regarding their potential importance as HRQoL predictors was available were entered into all models (shown in bold in Supplementary Figure 1): age, sex, socio-economic status, number of co-morbidities, time since diagnosis, stoma, BMI, physical activity, anxiety and depression scores, baseline fatigue and baseline HRQoL score of the specific domain. Additionally, in each of the 50 imputed datasets, other candidate predictors for which the evidence was considered weak-tomoderate or inconclusive [27] were tested for inclusion into the models by a backwards stepwise elimination procedure, using $P<0.1573$ as cut-off for inclusion based on Akaike's Information Criterion [44, 45]. Predictors were included in the final models when they were not eliminated from the models in $\geq 50 \%$ of the 50 imputed datasets [46]. Finally, regression coefficients from each imputed dataset were pooled using Rubin's rules [47].

Measures of discrimination, calibration, overall performance, and classification were determined for each final model for the separate HRQoL domains. Discriminative ability describes how well a model can distinguish between individuals with low vs. normal/high HRQoL based on estimated risks, as quantified by the area under the Receiver Operator Characteristic curve (AUC, with AUC >0.8 indicating good discrimination) [48]. Calibration is the agreement between predicted probabilities (risk) and observed relative frequencies (prevalence) of low HRQoL in the separate domains, as assessed by visual inspection of calibration plots showing agreement between predicted risk and observed prevalence of low HRQoL within deciles of predicted risk scores [49]. In addition, we used the Hosmer-Lemeshow goodness-of-fit test $(\mathrm{H}-\mathrm{L})$, with $P>0.05$ indicating adequate calibration. To assess overall model performance, Nagelkerke's $\mathrm{R}^{2}$ was determined as measure of predictive strength ranging between 0 and 1 with higher values indicating better performance, and Brier scores were determined as measures of model accuracy normally ranging between 0 and 0.25 with lower scores reflecting greater accuracy. Finally, for a range of predicted 
probabilities (10-80\%), sensitivity and specificity of the models were determined as measures of classification, with sensitivity reflecting the probability that low HRQoL is correctly predicted in persons actually having low HRQoL (i.e. percentage of true-positive predictions given low HRQoL), and specificity reflecting the probability that normal/high HRQoL is correctly predicted in persons actually having normal/high HRQoL (i.e. percentage of true-negative predictions given no low HRQoL). We defined optimal threshold probabilities for the separate models based on high sensitivity ( $>80 \%)$, as we considered false-negative predictions (i.e. misclassifying individuals with low HRQoL into the normal/high HRQoL group) more 'harmful' than false-positive predictions (i.e. misclassifying individuals with normal/high HRQoL into the low HRQoL group).

All final models were internally validated by bootstrapping using 1000 bootstrap samples to determine the degree of overfitting (i.e. models performing better in the development sample than in new samples consisting of other subjects) [44], yielding shrinkage factors for adjusting regression coefficients and adjusted model intercepts for incorporation into prediction formulas, and to assess optimism-corrected model performance measures [50, 51].

As sensitivity analyses, we reran the final models in the original non-imputed dataset to check if analyses yielded different conclusions after the multiple imputation as compared to complete-case analysis. Furthermore, we also performed backwards elimination procedures with less stringent $P$-values $(P<0.5)$ as cut-off for inclusion to assess whether relevant predictors were missed and affected model performance measures. In order to see the value of baseline HRQoL with regard to having low levels at the follow-up, we also ran models with only the respective baseline added, with the models excluding baseline, and compared the AUCs with the final models. All analyses were performed using $\mathrm{R}$ statistical software (R Foundation for Statistical Computing Platform 2016@, version 3.3.1). The Transparent Reporting of a multivariable prediction model for Individual Prognosis Or Diagnosis (TRIPOD) statement was used as guideline for analysis and reporting $[25,26]$.

\section{Results}

\section{Population characteristics}

Of the 1458 participants, 229 to 371 (16-25\%) were categorized into the low HRQoL groups for the different domains, with the majority having consistently low HRQoL (57-71\%, Table 1). Participants were on average 70 years of age and 5.1 years post-diagnosis, $43 \%$ was female, and 59 and $41 \%$ were diagnosed with colon or rectum cancer, respectively (Table 2). Complete data was available from 790 (54\%) participants, whereas 668 participants (46\%) had at least one missing value. Compared to participants
Table 2 Predictors measured at baseline and follow-up (T1) in the entire population in non-imputed dataset $(N=1458)$

\begin{tabular}{lll}
\hline & $N$ & $N(\%)$ \\
\hline Sociodemographic factors & & \\
Age (mean in years, SD) & 1458 & $70.0(9.3)$ \\
Sex (female) & 1458 & $624(42.8 \%)$ \\
Marital status (not married) & 1439 & $322(22.1 \%)$ \\
Work status (not working) & 1415 & $1166(80.0 \%)$ \\
Socio-economic status level & 1419 & \\
$\quad$ Low & & $263(18.0 \%)$ \\
$\quad$ Medium & & $595(40.8 \%)$ \\
High & & $561(38.5 \%)$
\end{tabular}

Clinical Factors

Number of comorbidities 1381

None

$351(24.1 \%)$

$\geq 2$

$429(29.4 \%)$

$601(41.2 \%)$

Time since diagnosis (mean in years, SD) $\quad 1458$

$5.1(0.1)$

Tumor site (colon vs. rectum)

$861(59.1 \%)$

Tumor stage

I

$446(30.6 \%)$

$512(35.1 \%)$

$418(28.7 \%)$

$41(2.8 \%)$

IV

Stoma present $(y / n)$

$309(21.2 \%)$

Chemotherapy $(\mathrm{y} / \mathrm{n})$

$431(29.6 \%)$

Radiotherapy $(\mathrm{y} / \mathrm{n})$

$478(32.8 \%)$

Stoma-related complaints (mean, SD)

$3.9(11.4)$

Pain (mean, SD)

$15.9(23.7)$

Micturition problems (mean, SD)

$21.7(17.6)$

Chemotherapy-related side effects (mean, SD)

$10.0(14.8)$

Gastro-intestinal complaints (mean, SD)

$47.2(51.8)$

Body composition and lifestyle factors

Body mass index (mean in $\mathrm{kg} / \mathrm{m}^{2}, \mathrm{SD}$ )

Waist circumference (mean in $\mathrm{cm}, \mathrm{SD})^{\text {a }}$

$26.7(4.1)$

Smoking $(y / n)$

$95.5(14.8)$

Alcohol consumption ${ }^{a}$

Non-drinker

Light-moderate drinker

Heavy drinker

Physical activity (adherence) ${ }^{a}$

Fruit and vegetables (non-adherence) ${ }^{a}$

Meat consumption (non-adherence) ${ }^{\text {a }}$

$\begin{array}{ll}1446 & 26.7(4.1) \\ 1198 & 95.5(14.8) \\ 1420 & 141(9.7 \%) \\ 1168 & \\ & 316(21.7 \%) \\ & 560(38.4 \%) \\ & 292(20.0 \%) \\ 1242 & 903(91.9 \%) \\ 1207 & 579(39.7 \%) \\ 1215 & 598(41.0 \%) \\ & \\ 1412 & 4.3(3.5) \\ 1419 & 4.3(3.5) \\ 1402 & 6.6(5.9) \\ 1409 & 7.6(5.9) \\ \end{array}$

Psychological factors

Anxiety symptoms (mean, SD)

Depressive symptoms (mean, SD)

Negative affectivity (mean, SD)

Social inhibition (mean, SD)

${ }^{\text {a }}$ Predictors measured at $\mathrm{T} 1$ 
with complete data, participants with incomplete data were more often female (48\% vs. $39 \%$ ), somewhat older (72 vs. 69 years), adhered less to physical activity guidelines $(60 \%$ vs. $80 \%)$, and had somewhat lower HRQoL scores (3-11\% more participants categorized into low HRQoL groups).

\section{Prediction model development and internal validation}

In the different prediction models for the seven separate HRQoL domains, 14 to 18 predictors were included in total, of which 12 predictors were entered into all models (or 11 for the model with fatigue as outcome) and 2 to 6 additional predictors were selected based on the backwards elimination procedure. Table 3 shows the intercepts and pooled regression coefficients of the predictors after correction for the shrinkage factors. Even though associations of individual predictors with the outcomes are not of primary importance when developing and evaluating performance of risk prediction models, optimism-corrected odds ratios are presented in Supplementary Table 1 to provide an indication of the magnitude and direction of the relations of each predictor with the separate HRQoL outcomes.

All model performance measures are shown in detail in Supplementary Table 2. Internal validation yielded shrinkage factors ranging between 0.89 and 0.91 for the separate models. The optimism-corrected AUC values ranged between 0.83 and 0.93 , which are also shown together with the ROC curves in Fig. 2. Nagelkerke's $R^{2}$ values ranged between 0.40 and 0.63 , and Brier scores between 0.09 and 0.15 . Calibration of the models was good, as indicated by calibration plots showing good agreement between actual and predicted probabilities for all models (Supplementary Figure 2). Additionally, all Hosmer-Lemeshow goodness-of-fit tests were nonsignificant for all HRQoL domains ( $P$-values ranging between 0.32 and 0.95$)$. Graphs with sensitivity and specificity plotted for the separate models across a range 10 to $80 \%$ predicted risk of low HRQoL showed that a sensitivity of $80 \%$ or higher was reached when predicted risks between 10 and 30\% were used a cut-off for a positive prediction, i.e. classification of an individual into the low HRQoL group based on the predicted risk score (Supplementary Figure 3). Overall, the prediction model with physical functioning as outcome was the model that showed the best performance.

\section{Sensitivity analyses}

Sensitivity analyses demonstrated that the final models were robust, as they performed similarly in the imputed and the original non-imputed datasets, yielding comparable AUC values (AUC range: 0.85-0.94, data not shown). In addition, AUC values also did not change when less stringent backward selection criteria
$(P<0.5)$ were used for model development (AUC range: $0.85-0.94$, data not shown). The AUCs of models were slightly smaller when they contained only baseline HRQoL (AUC range: 0.80-0.92), or without any baseline HRQoL (AUC range: $0.78-0.88$, as shown in Supplementary Table 3).

\section{Discussion}

Risk prediction models for seven HRQoL domains in long-term CRC survivors were developed and internally validated, containing a comprehensive set of evidencebased biopsychosocial predictors and showing good to excellent model performance. These models are ready for external validation in other cohorts of CRC survivors, who are for instance situated closer to diagnosis and treatment. This would be to evaluate whether they are generalizable and could be useful tools in oncology practice for identifying individual CRC survivors at risk of experiencing low HRQoL approximately one year after the moment of prediction. Thus, use of the prediction models can enable selection of high-risk individuals who might benefit from interventions aimed at improving or safeguarding their future HRQoL.

As the first important step in prediction research, this large-scale study has provided internally valid prediction models for estimating the 1-year risk of having low HRQoL. Firstly, these models have lifestyle and psychosocial predictors included that are selected based on evidence from previous association studies summarized in a systematic review [27]. When we ran the models with only the respective baseline HRQoL values, their AUCs were relatively high, confirming the prior expectation that baseline HRQoL alone is an important predictor that should be included in the models. Nevertheless, the AUCs increase when the other predictors are included, indicating improved predictions. Moreover, the table shows that the other predictors do have adequate predictive power, as shown by the AUC results of the models without baseline HRQoL. The associations between HRQoL and the specific predictors were not part of our scope, and therefore, we should be cautious with a causal interpretation of relations between predictors and outcomes based on prediction models. Even though the developed risk prediction models included partially overlapping predictors, the models for each of the seven HRQoL domains had their own unique features and contributions to the risk estimation. Moreover, low to moderate correlations observed among the HRQoL domains (Spearman's rho: 0.3-0.5; data not shown) indicated that the low HRQoL groups for the separate domains were not comprised of the same CRC survivors, reflecting that HRQoL is a multi-dimensional construct consisting of different aspects that are covered by the separate domains. 
Table 3 Regression coefficients of the included predictors of the seven prediction models for health-related quality of life (HRQoL), after internal validation and shrinkage

\begin{tabular}{|c|c|c|c|c|c|c|c|}
\hline & $\begin{array}{l}\text { Global quality } \\
\text { of life }\end{array}$ & $\begin{array}{l}\text { Cognitive } \\
\text { Functioning }\end{array}$ & $\begin{array}{l}\text { Emotional } \\
\text { Functioning }\end{array}$ & $\begin{array}{l}\text { Physical } \\
\text { Functioning }\end{array}$ & $\begin{array}{l}\text { Role } \\
\text { Functioning }\end{array}$ & $\begin{array}{l}\text { Social } \\
\text { Functioning }\end{array}$ & Fatigue $^{c}$ \\
\hline Intercept & -0.33 & 2.37 & -0.62 & 1.20 & -1.50 & -0.71 & -3.96 \\
\hline \multicolumn{8}{|l|}{ Included forced entry predictors ${ }^{a}$} \\
\hline Age (years) & 0.02 & 0.00 & 0.02 & 0.05 & 0.03 & 0.02 & 0.01 \\
\hline Sex (ref = male) & 0.27 & -0.16 & 0.11 & 0.42 & 0.15 & -0.02 & 0.20 \\
\hline \multicolumn{8}{|l|}{ Socio-economic status (ref = high) } \\
\hline Medium & 0.22 & 0.13 & 0.14 & -0.06 & 0.21 & 0.06 & -0.11 \\
\hline Low & 0.00 & 0.17 & -0.03 & -0.19 & -0.05 & -0.20 & -0.42 \\
\hline \multicolumn{8}{|l|}{ Number of co-morbidities (ref = none) } \\
\hline 1 & -0.14 & 0.17 & 0.06 & 0.18 & 0.06 & 0.22 & 0.12 \\
\hline$\geq 2$ & 0.10 & -0.10 & 0.23 & 0.49 & 0.32 & 0.47 & 0.29 \\
\hline Time since diagnosis (years) & 0.02 & -0.04 & 0.03 & -0.01 & -0.03 & -0.01 & 0.00 \\
\hline Stoma presence $(r e f=n o)$ & -0.18 & 0.00 & 0.11 & -0.02 & 0.40 & 0.28 & -0.21 \\
\hline Body mass index $\left(\mathrm{kg} / \mathrm{m}^{2}\right)$ & 0.02 & -0.01 & -0.05 & 0.01 & -0.01 & 0.00 & -0.01 \\
\hline Physical activity (ref = non-adherence) & -0.36 & 0.02 & -0.06 & -0.64 & -0.44 & -0.25 & -0.45 \\
\hline Anxiety symptom score & 0.02 & -0.01 & 0.14 & 0.03 & 0.05 & -0.01 & 0.04 \\
\hline Depressive symptom score & 0.08 & 0.07 & 0.03 & 0.03 & 0.04 & 0.08 & 0.05 \\
\hline Baseline fatigue & 0.01 & 0.01 & 0.01 & 0.01 & 0.01 & 0.01 & 0.05 \\
\hline Baseline HRQoL (specific per domain) & -0.05 & -0.06 & -0.04 & -0.10 & -0.03 & -0.04 & - \\
\hline \multicolumn{8}{|c|}{ Included predictors based on backwards selection ${ }^{\mathbf{b}}$} \\
\hline Chemotherapy (ref = no) & 0.25 & & & & & -0.35 & \\
\hline Radiotherapy (ref = no) & 0.27 & & & & & & \\
\hline \multicolumn{8}{|l|}{ Tumour stage $($ ref = stage I) } \\
\hline Stage II & & & & & & -0.11 & \\
\hline Stage III & & & & & & 0.52 & \\
\hline Stage IV & & & & & & 0.50 & \\
\hline Working status (ref = no) & & & 0.48 & & & & \\
\hline Smoking $(r e f=n o)$ & 0.36 & & 0.41 & 0.66 & & 0.56 & 0.81 \\
\hline Social inhibition score & & & -0.03 & & 0.02 & & \\
\hline Negative affectivity score & & 0.03 & 0.06 & & & 0.04 & \\
\hline Micturition score & & 0.01 & & & & & \\
\hline Chemotherapy side effects score & 0.01 & & 0.01 & & & 0.01 & 0.01 \\
\hline Stoma complaints score & 0.01 & & & 0.02 & & & 0.02 \\
\hline Gastrointestinal complaints sum score & & & & & & 0.01 & 0.00 \\
\hline Pain score & 0.01 & & & & 0.01 & & 0.01 \\
\hline Meat consumption adherence (ref = yes) & & & 0.22 & & & & \\
\hline
\end{tabular}

Footnotes:

a Twelve candidate predictors were forced into each model, as there was strong evidence for their association with HRQoL in a systematic review [19]

${ }^{b}$ Candidate predictors for which moderate or weak evidence was found, were selected with backwards selection procedures using Akaike's Information Criterion $(p<0.1573)$. The following candidate predictors were not included in any of the models: tumor localization, marital status, fruit and vegetable consumption, alcohol consumption and waist circumference

c Regression coefficients display the In (odds) change in outcome, but no standard errors could be calculated after shrinkage; Formula for the probability of having low HRQoL $=1 /(1+$ exp.[- Linear predictor]);

Linear predictor $=$ intercept + sum of (predictor ${ }^{*}$ regression coefficient) 
A) Global quality of life

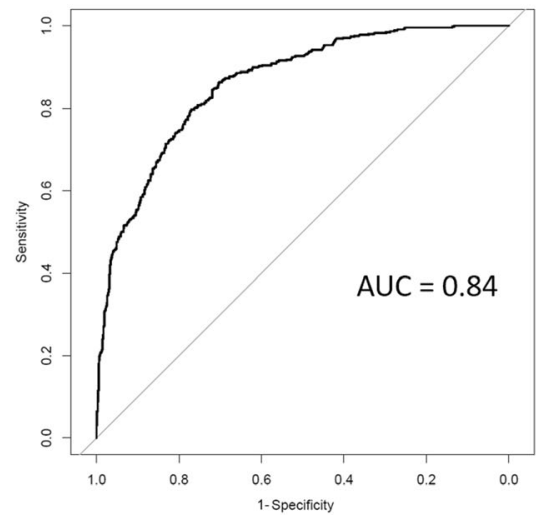

B) Cognitive functioning
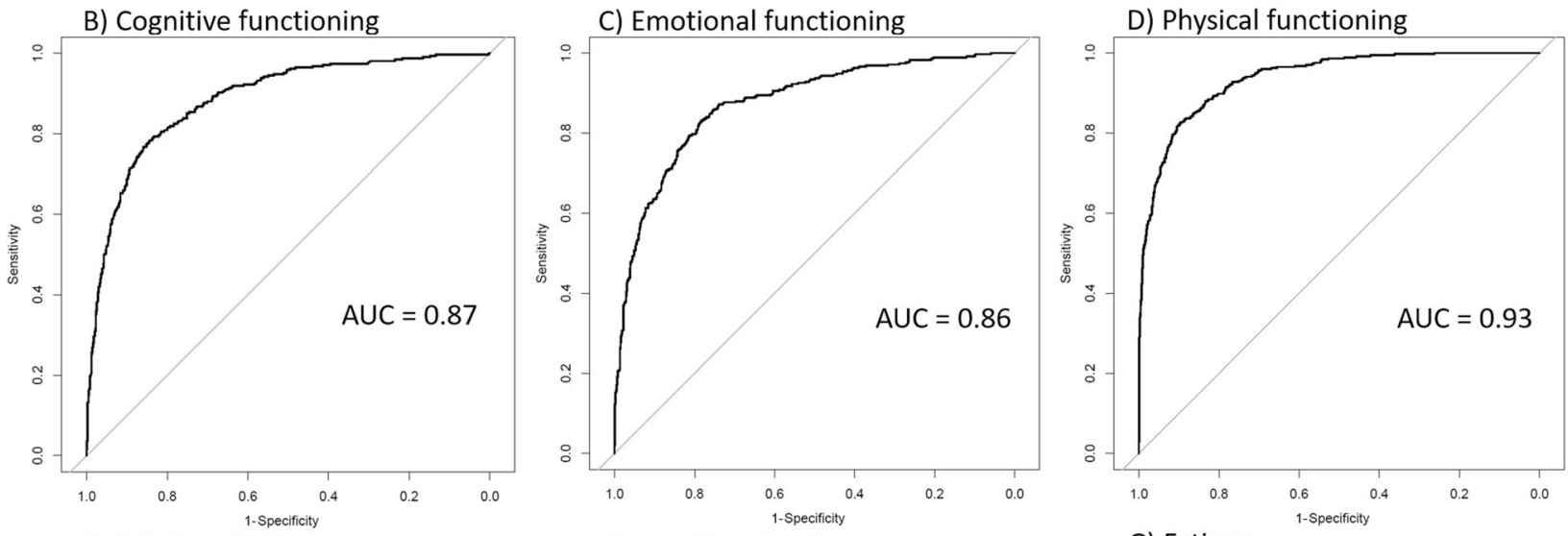

E) Role functioning

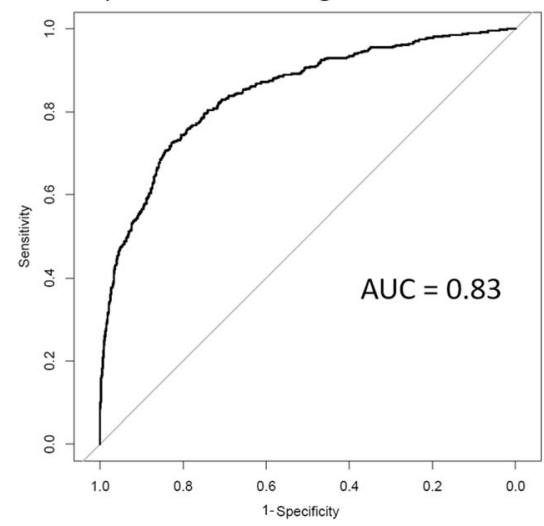

F) Social functioning

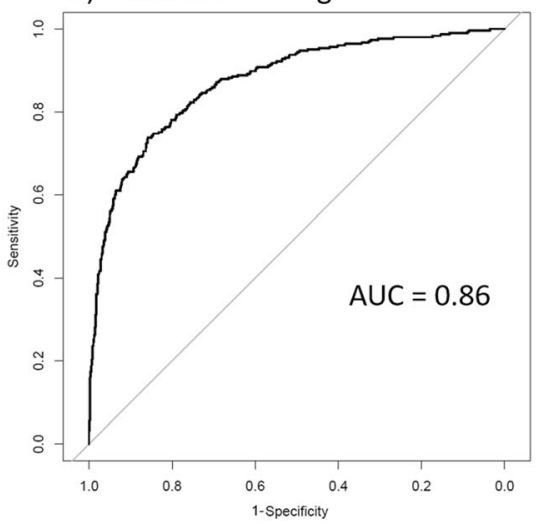

G) Fatigue

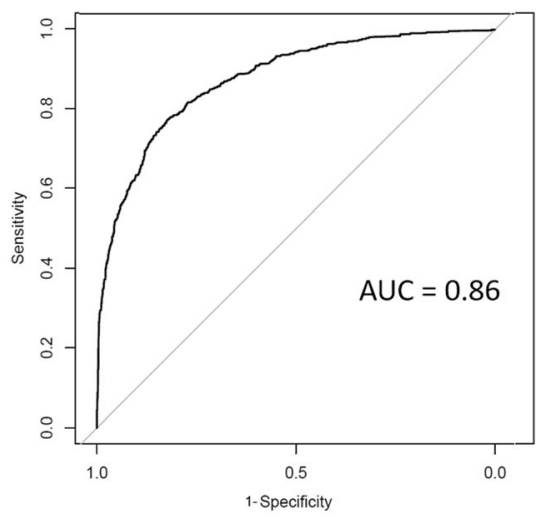

Fig. 2 For each health-related quality of life domain we show the area under the Receiver Operator Characteristic curve (AUC, with AUC $>0.8$ indicating good discrimination)

The predictive power of all 7 models was good to excellent. The models were found to generate accurate risk predictions that enabled good discrimination between individual CRC survivors who did or who did not experience low HRQoL scores in the future. Further, it was found that optimal probability thresholds for good classification of low vs. normal/high HRQoL based on predicted risks mostly ranged between 10 and $30 \%$. If predicted risks within this range were used as cut-off for positive predictions (i.e. classification of an individual survivor as being at risk of low HRQoL), the sensitivities of the models were $>80 \%$ which is considered high. We preferred a high sensitivity of the models over a high specificity, because we did not want to misclassify many survivors with low HRQoL (false-negatives) who could benefit from interventions targeted at improving their future HRQoL. We accepted lower specificity of the models (i.e. increased chance of false-positive 
predictions) since we deemed providing unnecessary HRQoL interventions, which are not invasive or hazardous, less problematic than not providing necessary HRQoL interventions.

For the current study, long-term CRC survivors participating in an ongoing prospective cohort study were selected. Two third of the survivors classified into the low HRQoL group at study follow-up also had low HRQoL scores at baseline, indicative of a consistently low level of HRQoL. Nevertheless, a substantial percentage of the CRC survivors showed a clinically relevant deterioration of HRQoL scores over the approximately 1 -year study period, which is rather striking when considering that the CRC survivors were on average five years after diagnosis. Larger changes in HRQoL are expected closer to diagnosis and treatment [52], which may be a more relevant time frame for prediction and taking preventive action. Therefore, the next step should be to externally validate the developed models in other CRC survivor populations to determine whether their predictive abilities are transferable to a more immediate post-treatment time frame. Subsequently, the benefit of these models should also be evaluated in so-called clinical impact studies to assess whether risk prediction is of added value and can contribute to improving HRQoL outcomes in oncology practice. This final and important step of prediction research is often overlooked. For instance, several prediction models have been developed, and to a lesser extent externally validated, for estimating probabilities of survival in CRC patients to be used when considering different treatment options [18-20]. One recently published prediction model for survival has even presented an online tool for use in clinical practice during the treatment phase [20]. However, none of these previously developed models for survival have been evaluated in clinical impact studies to assess whether their application actually can improve survival through improved tailoring of treatments.

The present study has several strengths, including its large sample size, high response rate, and longitudinal design. In addition, sophisticated statistical methods were used that are currently recommended in the field of prediction modelling, such as multiple imputation and bootstrapping [26]. Furthermore, all predictors were selected from the literature based on previous evidence [27], thereby emphasizing theorydriven instead of data-driven predictor selection. Moreover, our study is novel as, to the best of our knowledge, no prediction models for estimating future HRQoL in CRC survivors after treatment are currently available. Both clinicians and CRC survivors could benefit from future implementation of such models in the form of, for example, online calculators or as add-ons to existing lifestyle and clinical guidelines (e.g. from WCRF/AICR [35, 53] and American Cancer Society [1]) that focus mostly on cancer prevention and survival but less on HRQoL.

Next to its strengths, the study also has some limitations. First, as already pointed out, the study participants were long-term CRC survivors on average five years after diagnosis, therefore representing a population that probably had relatively stabilized HRQoL. As mentioned, future external validation of these models is warranted in cohorts closer to diagnosis and treatment, when larger changes are expected in HRQoL. Second, we dichotomized the continuous HRQoL outcomes for ease of interpretation and risk estimation, which may have led to loss of information. The classification of survivors in the low and normal/high HRQoL groups at study follow-up was determined based on the mean HRQoL scores at the study baseline, and therefore population-dependent. However, we also incorporated previously reported cut-offs for HRQoL deteriorations to make the classifications more clinically relevant and generalizable [30]. Third, we defined the low HRQoL group as having low HRQoL at study follow-up, thereby not distinguishing between individuals with consistently low HRQoL or with deteriorated HRQoL over time. Though both groups of individuals would be eligible for interventions aimed at safeguarding their HRQoL, future studies could elaborate on the longitudinal course of HRQoL and on possible different characteristics of individuals at risk of having constant low levels of HRQoL or deteriorating levels of HRQoL. Fourth, regardless of the large sample size, models had a range of 8 to 13 events per predictor, and some models had less than the recommended $\geq 10$ events per predictor for model development and less than 250 events for the internal validation [26], which might have impacted the stability of the performance measures. In a recently published tool to assess risk of bias in prediction model studies, Moons et al. state that development studies should have more than 20 events per predictor, and more than 100 events for the validations [54]. Moreover, we did not apply any interaction terms at the development of these models for terms like age or BMI. Most of the included participants were older (mean age $=70.0$ years; $\mathrm{SD}=9.3$ ), as most CRC survivors are in the practice too. Also, BMI may be interesting to look at when we distinguish between underweight and overweight, but also there was not that much difference (mean $\mathrm{BMI}=26.7 ; \mathrm{SD}=$ 4.1). We are aware of the multiple techniques available for prediction modelling in addition to regression analyses (e.g., machine learning techniques such as random forests, neural networks), but we now used regression modelling, as this is how the majority of the models is developed. Moreover, advanced techniques are not always superior [55], and it remains transparent, reproducible and understandable for clinicians and researchers. Lastly, imputation 
of missing values might have introduced bias if the missings were not random. Although this assumption is untestable, multiple imputation was used as the currently recommended strategy for imputing missing data with the least risk of bias [26, 54].

\section{Conclusion}

To our knowledge, this is the first study that developed and internally validated prediction models for HRQoL in CRC survivors, focusing on estimating the 1-year risk of low HRQoL in multiple domains (global QoL; cognitive, emotional, physical, role, and social functioning; and fatigue). The models showed good to excellent predictive performance for identifying CRC survivors who are at increased risk of experiencing low HRQoL in the future and who are eligible for preventive interventions. The included set of biopsychosocial predictors, of which several are modifiable, have been significantly associated with HRQoL in CRC survivors in the literature. In the future, external validation and a clinical impact evaluation are needed before these models should be used for decision making. As there is often a lack of time during oncological consultations to discuss HRQoL problems, prediction models can enhance efficient communication with patients and shared decision-making. The developed models are important as a first step towards future implementation of risk prediction tools in oncology practice specifically aimed at the HRQoL of the growing population of CRC survivors.

\section{Supplementary information}

Supplementary information accompanies this paper at https://doi.org/10. 1186/s12911-020-1064-9.

Additional file 1: Supplemental Figure S1. Predictors mapped across domains of the World Health Organization's International Classification of Functioning, Disability and Health (WHO-ICF) framework, and selected for the prediction models based on previous evidence: 12 fixed predictors entered into all models (in bold with arrows) and 18 candidate predictors selected for backwards elimination. Some candidate predictors were measured at T1 instead of T0; this is indicated between brackets.

Additional file 2: Supplemental Figure S2. Calibration plots for seven health-related quality of life (HRQoL) domains.

Additional file 3: Supplemental Figure S3. Classification measures of the models for the seven health-related quality of life (HRQoL) domains with various threshold probabilities (10-80\%): sensitivity (probability of true-positive prediction given low HRQoL; black line) and specificity (probability of true-negative prediction given no low HRQoL; dotted line). Grey boxes highlight threshold probabilities that correspond to sensitivity $>80 \%$.

Additional file 4: Supplemental Table S1. Odds ratios of included predictors of the seven prediction models for health-related quality of life (HRQOL) after internal validation.

Additional file 5: Supplemental Table S2. Model performance measures of the seven prediction models for health-related quality of life. Performance measures of the original models and the models after internal validation are presented.
Additional file 6: Supplemental Table S3. Sensitivity analyses of the seven prediction models for health-related quality of life (HRQOL), with only respective baseline HRQoL values, without baseline HRQoL and with the complete models.

\section{Abbreviations}

AUC: Area under the Receiver Operator Characteristic curve; BMI: Body mass index; CRC: Colorectal cancer; DHD-FFQ: Dutch Healthy Diet-Food Frequency Questionnaire; DS-14: Dutch 14-item Type D Personality Scale; EORTC QLQC30: European Organization for Research and Treatment of Cancer Quality of life Questionnaire - Core 30; HADS: Hospital Anxiety and Depression Scale; HL: Hosmer-Lemeshow goodness-of-fit test; HRQoL: Health-related quality of life; MID: Minimally important deteriorations; MVPA: Moderate-to-vigorous intensity physical activity; PROFILES: Patient Reported Outcomes Following Initial Treatment and Long-Term Evaluation of Survivorship; QoL: Quality of life; SES: Socio-economic status; SQUASH: Short QUestionnaire to ASsess Health-enhancing physical activity; TRIPOD: Transparent Reporting of a multivariable prediction model for Individual Prognosis Or Diagnosis; WCRF/ AICR: World Cancer Research Fund/American Institute for Cancer Research; WHO-ICF: World Health Organization's International Classification of Functioning, Disability and Health

\section{Acknowledgements}

Not applicable.

\section{Authors' contributions}

MJLB, MPW, SMJVK and DR have contributed to the conception and design of the work. DR, SMJVK, LJS and MJLB have done the analysis and interpretation of the data. DR, SMJVK, HH, LJS, and MJLB have substantively revised it. All authors have approved the submitted version (and any substantially modified version that involves the author's contribution to the study).

\section{Funding}

This work was supported by a grant from the Alpe d'HuZes Foundation within the research program "Leven met kanker" of the Dutch Cancer Society (Grant number UM-2012-5653), and also partly by the Kankeronderzoekfonds Limburg as part of Health Foundation Limburg (Grant number 00005739). These funders only funded the design of the paper, and played no role in the data collection, analysis, interpretation of data or in the writing of the manuscript.

\section{Availability of data and materials}

The datasets used and/or analysed during the current study are available from the corresponding author on reasonable request.

\section{Ethics approval and consent to participate}

All procedures performed in studies involving human participants were approved by the medical ethical committee of the Maxima Medical Center in Veldhoven, The Netherlands (number 0822) and with the 1964 Helsinki declaration and its later amendments or comparable ethical standards. All patients signed informed consent.

\section{Consent for publication}

Not applicable.

\section{Competing interests}

The authors declare that they have no conflict of interest.

\section{Author details}

${ }^{1}$ Department of Epidemiology, GROW - School for Oncology and Developmental Biology, Maastricht University, P. Debyeplein 1, 6200, MD, Maastricht, the Netherlands. ${ }^{2}$ Department of Medical and Clinical Psychology, CoRPS - Center of Research on Psychology in Somatic diseases, Tilburg University, Warandelaan 2, 5037, AB, Tilburg, the Netherlands. ${ }^{3}$ Clinical Epidemiology and Medical Technology Assessment, Maastricht University Medical Centre+, P. Debyelaan 25, PO Box 5800, Maastricht 6202, AZ, the Netherlands. ${ }^{4}$ Netherlands Comprehensive Cancer Organisation (IKNL), Godebaldkwartier 419, 3511, DT, Utrecht, the Netherlands. ${ }^{5}$ Division of Human Nutrition, Wageningen University \& Research, Stippeneng 4, 6708, WE, Wageningen, the Netherlands. ${ }^{6}$ Department of Public Health Sciences, 
Penn State Cancer Institute, 500 University, Hershey, PA 17033, USA. 7 Department of Epidemiology, CAPHRI School for Public Health and Primary Care, Faculty of Health, Medicine and Life Sciences, Maastricht University, P. Debyeplein 1, 6200, MD, Maastricht, the Netherlands. ${ }^{8}$ Department of Surgery, Maastricht University Medical Centre, P. Debyelaan 25, 6229, HX, Maastricht, the Netherlands. ${ }^{9}$ Department of Psychosocial Oncology and Epidemiology, Netherlands Cancer Institute, Plesmanlaan 121, 1066, CX, Amsterdam, the Netherlands.

\section{Received: 30 September 2019 Accepted: 23 February 2020} Published online: 12 March 2020

\section{References}

1. El-Shami K, Oeffinger KC, Erb NL, Willis A, Bretsch JK, Pratt-Chapman ML, et al. American Cancer Society colorectal Cancer survivorship care guidelines. CA Cancer J Clin. 2015:65(6):428-55.

2. Shapiro CL. Cancer survivorship. N Engl J Med. 2018;379(25):2438-50.

3. Bray F, Ferlay J, Soerjomataram I, Siegel RL, Torre LA, Jemal A. Global cancer statistics 2018: GLOBOCAN estimates of incidence and mortality worldwide for 36 cancers in 185 countries. CA Cancer J Clin. 2018;68(6):394-424.

4. Marventano S, Forjaz M, Grosso G, Mistretta A, Giorgianni G, Platania A, et al. Health related quality of life in colorectal cancer patients: state of the art. BMC Surg. 2013; 13(Suppl 2):S15

5. Jansen $L$, Koch $L$, Brenner $H$, Arndt V. Quality of life among long-term $(>/=5$ years) colorectal cancer survivors--systematic review. Eur J Cancer. 2010; 46(16):2879-88.

6. Arndt V, Koch-Gallenkamp L, Jansen L, Bertram H, Eberle A, Holleczek B, et al. Quality of life in long-term and very long-term cancer survivors versus population controls in Germany. Acta Oncol. 2017;56(2):190-7.

7. Moug SJ, Bryce A, Mutrie N, Anderson AS. Lifestyle interventions are feasible in patients with colorectal cancer with potential short-term health benefits: a systematic review. Int J Color Dis. 2017;32(6):765-75.

8. Hawkes AL, Pakenham Kl, Chambers SK, Patrao TA, Courneya KS. Effects of a multiple health behavior change intervention for colorectal cancer survivors on psychosocial outcomes and quality of life: a randomized controlled trial. Ann Behav Med. 2014;48(3):359-70.

9. Mishra SI, Scherer RW, Snyder C, Geigle P, Gotay C. Are exercise programs effective for improving health-related quality of life among cancer survivors? A systematic review and meta-analysis. Oncol Nurs Forum. 2014; 41(6):E326-42

10. Mishra SI, Scherer RW, Snyder C, Geigle PM, Berlanstein DR, Topaloglu O. Exercise interventions on health-related quality of life for people with cancer during active treatment. Cochrane Database Syst Rev. 2012;8: CD008465.

11. Turner RR, Steed L, Quirk H, Greasley RU, Saxton JM, Taylor SJ, et al. Interventions for promoting habitual exercise in people living with and beyond cancer. Cochrane Database Syst Rev. 2018;9:CD010192.

12. Mosher CE, Winger JG, Given BA, Shahda S, Helft PR. A systematic review of psychosocial interventions for colorectal cancer patients. Support Care Cancer. 2017:25(7):2349-62.

13. Son $\mathrm{H}$, Son $\mathrm{YJ}$, Kim H, Lee $\mathrm{Y}$. Effect of psychosocial interventions on the quality of life of patients with colorectal cancer: a systematic review and meta-analysis. Health Qual Life Outcomes. 2018;16(1):119.

14. Sales PM, Carvalho AF, McIntyre RS, Pavlidis N, Hyphantis TN. Psychosocial predictors of health outcomes in colorectal cancer: a comprehensive review. Cancer Treat Rev. 2014:40(6):800-9.

15. Glavic Z, Galic S, Krip M. Quality of life and personality traits in patients with colorectal cancer. Psychiatr Danub. 2014;26(2):172-80.

16. Gray NM, Hall SJ, Browne S, Macleod U, Mitchell E, Lee AJ, et al. Modifiable and fixed factors predicting quality of life in people with colorectal cancer. Br J Cancer. 2011;104(11):1697-703.

17. Steyerberg EW, Vergouwe $Y$. Towards better clinical prediction models: seven steps for development and an ABCD for validation. Eur Heart J. 2014;35(29):1925-31.

18. Kawai K, Sunami E, Yamaguchi H, Ishihara S, Kazama S, Nozawa H, et al. Nomograms for colorectal cancer: a systematic review. World J Gastroenterol. 2015;21(41):11877-86.

19. Engelhardt EG, Revesz D, Tamminga HJ, Punt CJA, Koopman M, OnwuteakaPhilipsen BD, et al. Clinical usefulness of tools to support decision-making for palliative treatment of metastatic colorectal Cancer: a systematic review. Clin Colorectal Cancer. 2017.
20. Hippisley-Cox J, Coupland C. Development and validation of risk prediction equations to estimate survival in patients with colorectal cancer: cohort study. BMJ. 2017;357:j2497.

21. Marventano S, Grosso G, Mistretta A, Bogusz-Czerniewicz M, Ferranti R, Nolfo F, et al. Evaluation of four comorbidity indices and Charlson comorbidity index adjustment for colorectal cancer patients. Int J Color Dis. 2014;29(9):1159-69.

22. Hendriksen JM, Geersing GJ, Moons KG, de Groot JA. Diagnostic and prognostic prediction models. J Thromb Haemost. 2013;11(Suppl 1):129-41.

23. Hingorani AD, Windt DA, Riley RD, Abrams K, Moons KG, Steyerberg EW, et al. Prognosis research strategy (PROGRESS) 4: stratified medicine research. BMJ. 2013;346:e5793.

24. Collins GS, Reitsma JB, Altman DG, Moons KG. Transparent reporting of a multivariable prediction model for individual prognosis or diagnosis (TRIPOD): the TRIPOD statement. Br J Surg. 2015;102(3):148-58.

25. Collins GS, Reitsma JB, Altman DG, Moons KG. Transparent reporting of a multivariable prediction model for individual prognosis or diagnosis (TRIPOD): the TRIPOD statement. Ann Intern Med. 2015;162(1):55-63.

26. Moons KG, Altman DG, Reitsma JB, loannidis JP, Macaskill P, Steyerberg EW, et al. Transparent reporting of a multivariable prediction model for individual prognosis or diagnosis (TRIPOD): explanation and elaboration. Ann Intern Med. 2015;162(1):W1-73.

27. Bours MJ, van der Linden BW, Winkels RM, van Duijnhoven FJ, Mols F, van Roekel EH, et al. Candidate predictors of health-related quality of life of colorectal Cancer survivors: a systematic review. Oncologist. 2016;21(4):433-52.

28. van de Poll-Franse LV, Horevoorts N, van Eenbergen M, Denollet J, Roukema JA, Aaronson NK, et al. The patient reported outcomes following initial treatment and long term evaluation of survivorship registry: scope, rationale and design of an infrastructure for the study of physical and psychosocial outcomes in cancer survivorship cohorts. Eur J Cancer. 2011:47(14):2188-94.

29. Aaronson NK, Ahmedzai S, Bergman B, Bullinger M, Cull A, Duez NJ, et al. The European Organization for Research and Treatment of Cancer QLQ-C30: a quality-of-life instrument for use in international clinical trials in oncology. J Natl Cancer Inst. 1993:85(5):365-76.

30. Cocks K, King MT, Velikova G, de Castro G Jr, Martyn St-James M, Fayers PM, et al. Evidence-based guidelines for interpreting change scores for the European organisation for the research and treatment of Cancer quality of life questionnaire Core 30. Eur J Cancer. 2012:48(11):1713-21.

31. van Duijn CK, I. Sociaal-economische status indicator op postcode niveau (Socioeconomic status indicator on zip code level). Maandstatistiek van de bevolking. 2002;50:32-5.

32. Sangha O, Stucki G, Liang MH, Fossel AH, Katz JN. The self-administered comorbidity questionnaire: a new method to assess comorbidity for clinical and health services research. Arthritis Rheum. 2003;49(2):156-63.

33. Sprangers MA, te Velde A, Aaronson NK. The construction and testing of the EORTC colorectal cancer-specific quality of life questionnaire module (QLQCR38). European Organization for Research and Treatment of Cancer study group on quality of life. Eur J Cancer. 1999;35(2):238-47.

34. Jones JM, Olson K, Catton P, Catton CN, Fleshner NE, Krzyzanowska MK et al. Cancer-related fatigue and associated disability in post-treatment cancer survivors. J Cancer Surviv. 2016;10(1):51-61.

35. World Cancer Research Fund / American Institute for Cancer Research. Food, nutrition, physical activity, and the prevention of cancer: a global perspective. Washington DC: AICR; 2007.

36. Wendel-Vos GC, Schuit AJ, Saris WH, Kromhout D. Reproducibility and relative validity of the short questionnaire to assess health-enhancing physical activity. J Clin Epidemiol. 2003;56(12):1163-9.

37. Ainsworth BE, Haskell WL, Leon AS, Jacobs DR Jr, Montoye HJ, Sallis JF, et al. Compendium of physical activities: classification of energy costs of human physical activities. Med Sci Sports Exerc. 1993;25(1):71-80.

38. van Lee L, Feskens EJ, Meijboom S, Hooft van Huysduynen EJ, van't Veer P, de Vries $\mathrm{JH}$, et al. Evaluation of a screener to assess diet quality in the Netherlands. Br J Nutr. 2016;115(3):517-26.

39. Zigmond AS, Snaith RP. The hospital anxiety and depression scale. Acta Psychiatr Scand. 1983;67(6):361-70.

40. Denollet J. DS14: standard assessment of negative affectivity, social inhibition, and type D personality. Psychosom Med. 2005;67(1):89-97.

41. Husson O, Vissers PA, Denollet J, Mols F. The role of personality in the course of health-related quality of life and disease-specific health status among colorectal cancer survivors: a prospective population-based study from the PROFILES registry. Acta Oncol. 2015;54(5):669-77. 
42. van Buuren S. Package 'mice' 2017. Available from: https://cran.r-project.org/ web/packages/mice/index.html.

43. Harrell FE, Jr. Package 'rms' 2017. Available from: https://cran.r-project.org/ web/packages/rms/rms.pdf.

44. Steyerberg EW. Clinical Prediction Models. A Practical Approach to Development, Validation, and Updating; 2009.

45. Harrell FE Jr. Regression Modeling Strategies. With Applications to Linear Models, Logistic and Ordinal Regression, and Survival Analysis. 2nd ed. Switzerland: Springer International Publishing AG; 2015. ISBN 978-3-31919424-0.

46. Vergouwe Y, Royston P, Moons KG, Altman DG. Development and validation of a prediction model with missing predictor data: a practical approach. J Clin Epidemiol. 2010;63(2):205-14.

47. Rubin DB. Multiple imputation multiple imputation for nonresponse in surveys. Canada: Wiley; 1987. ISBN 0-471-08705-X.

48. Hanley JA, McNeil BJ. The meaning and use of the area under a receiver operating characteristic (ROC) curve. Radiology. 1982;143(1):29-36.

49. Hosmer DWL. A goodness-of-fit test for the multiple logistic regression model Communications in Statistics, vol. A10; 1980. p. 1043-69.

50. Steyerberg EW, Bleeker SE, Moll HA, Grobbee DE, Moons KG. Internal and external validation of predictive models: a simulation study of bias and precision in small samples. J Clin Epidemiol. 2003;56(5):441-7.

51. Steyerberg EW, Harrell FE Jr, Borsboom GJ, Eijkemans MJ, Vergouwe Y, Habbema JD. Internal validation of predictive models: efficiency of some procedures for logistic regression analysis. J Clin Epidemiol. 2001;54(8):774-81.

52. Cabilan CJ, Hines S. The short-term impact of colorectal cancer treatment on physical activity, functional status and quality of life: a systematic review. JBI Database System Rev Implement Rep. 2017;15(2):517-66.

53. Diet N. Physical activity and Cancer: a global perspective; 2018.

54. Moons KGM, Wolff RF, Riley RD, Whiting PF, Westwood M, Collins GS, et al. PROBAST: a tool to assess risk of Bias and applicability of prediction model studies: explanation and elaboration. Ann Intern Med. 2019;170(1):W1-W33.

55. Christodoulou E, Ma J, Collins GS, Steyerberg EW, Verbakel JY, Van Calster B. A systematic review shows no performance benefit of machine learning over logistic regression for clinical prediction models. J Clin Epidemiol. 2019; 110:12-22.

\section{Publisher's Note}

Springer Nature remains neutral with regard to jurisdictional claims in published maps and institutional affiliations.

Ready to submit your research? Choose BMC and benefit from:

- fast, convenient online submission

- thorough peer review by experienced researchers in your field

- rapid publication on acceptance

- support for research data, including large and complex data types

- gold Open Access which fosters wider collaboration and increased citations

- maximum visibility for your research: over $100 \mathrm{M}$ website views per year

At BMC, research is always in progress.

Learn more biomedcentral.com/submissions 